\title{
Treatment of Complex Aorta Diseases by Multilayer Stents
}

Mohamed Nadjib Bouayed*, Leila Bouziane, Abderrahmane Sekkal and Sid Ahmed Benaroussi

Vascular Surgery Department, University Hospital, Hai sabah 31000 Oran, Algeria

*Corresponding author: Mohamed Nadjib Bouayed, Vascular Surgery Department, University Hospital, Hai sabah 31000 Oran, Algeria, Tel: 04142141234185; E-mail: bouayed_mohamed@yahoo.fr

Received date: April 22, 2016; Accepted date: June 15, 2016; Published date: June 20, 2016

Copyright: (c) 2016 Bouayed MN, et al. This is an open-access article distributed under the terms of the Creative Commons Attribution License, which permits unrestricted use, distribution, and reproduction in any medium, provided the original author and source are credited.

\begin{abstract}
:
Purpose: The aim of our study was to evaluate the role of multilayer stents (MLS) in the therapeutic arsenal of complex aortic pathologies for frail patients combining several comorbidities that are initially objected to the conventional surgical treatment.
\end{abstract}

Materials and methods: Since March 2012, we conducted a monocentric prospective study on a series of 41 procedures, performed on 41 aortic lesion locations in 38 patients treated with multilayer stents. The series was divided into four groups. The first group included 21 cases: 20 thoracoabdominal aneurysms and one aneurysm of the entire descending thoracic aorta. The second group included 7 cases of juxta/infra renal aneurysms, the third group included 5 cases of false aneurysms and the fourth group included 8 cases of aortic dissecting hematoma (first subgroup: 6 cases) or extended dissections (second subgroupe: 2 cases). Three patients were treated during the same operation for a double aneurysm location (thoracic and abdominal in 2 cases and aortic arch and thoracic aorta in the 3 rd case).

Results: The series included 25 men and 13 women with an average age of 63 years (40-84 years). The procedures were performed under local anesthesia in 3 cases, loco regional in 19 cases and general anesthesia in 16 cases. The average hospital stay was 7 days (4-14 days). The technical placement of the MLS was successful for the 41 procedures. No paraplegia, no stroke and no mesenteric ischemia occurred. Three complications related to the surgical approach were treated surgically with success. Two patients had post-operative renal failure for which one required dialysis. The 30-day mortality in relation to the aneurysm was $3 \%$ (one case of rupture in the first postoperative day) and unrelated to the aneurysm $3 \%$ (one case of massive myocardial infarction after the procedure). For an average follow up of 12 months (1-20 months) mortality unrelated to the aneurysm was $25 \%(9$ deaths) and $0 \%$ in relation to the aneurysm. Among the 38 remaining aortic lesions treated and followed, the aneurismal sac was not feeded in 14 cases (63\%) with 6 out of 20 cases in group 1(30\%), 3 out of 6 cases in group $2(50 \%), 3$ out of 4 cases in group $3(75 \%)$ and finally 2 out of 6 cases in the first subgroup of group $4(33 \%)$. The aneurismal sac is not feeded in 19 cases $(50 \%)$, including 14 out of 20 cases in group $1(70 \%), 2$ out of 6 cases in group $2(33 \%), 1$ out of 4 cases in the group 3(25\%) and 2 out of 6 cases in the first subgroup of group $4(33 \%)$. Two cases of extensive dissections treated remained stable without aneurismal changes. Three cases are recent: 1 out of 6 cases in group $2(17 \%)$ and 2 out of $6(33 \%)$ in the first subgroup of group $4(33 \%)$. Visceral arteries and supraaortic trunks remained patent in all the 38 cases.

Conclusion: MLS may be an option treatment for dissections and complex aortic aneurysms in frail patients with high surgical risk. Our results showed that better results are obtained for false aneurysms and true aortic aneurysms without involvement of significant side branches.

Keywords: Aneurysm; False aneurysm; Aortic dissection; Aorta; Multilayer flow modulators; Multilayer stents; Visceral arteries; Aortic hematoma

\section{Introduction}

Thoraco-abdominal aortic aneurysms (TAAA) are difficult to treat especially those interrelated to the visceral arteries or the supra-aortic trunks and aortic dissections (AD). Conventional surgery, directed most often to frail patients, is burdened with a significant morbidity and mortality. Given the difficulty of the surgical treatment and the severity of its complications, endovascular treatment continues to develop. Fenestrated and branched endoprografts (FBE) are thriving and are being evaluated.
Could Multilayer Stent (MLS) or Multilayer Flow Modulator (MFM) be a solution for the treatment of these complex aortic pathologies? What is the concept of the Multilayer Stent?

MLS is a self-expanding stent. It consists of many cobalt alloy wire, braided in several interconnected layers (Figure 1). In vitro [1], in vivo [2] and computerized studies showed that after the insertion of a MLS, vortex speed [3] in the aneurismal sac decreases and turbulent flows turn into laminar flows [3]. These two phenomena result in a decrease in pressure in the aneurysm and thus a reduction of wall shear stress with consequent protection against walls rupture. 


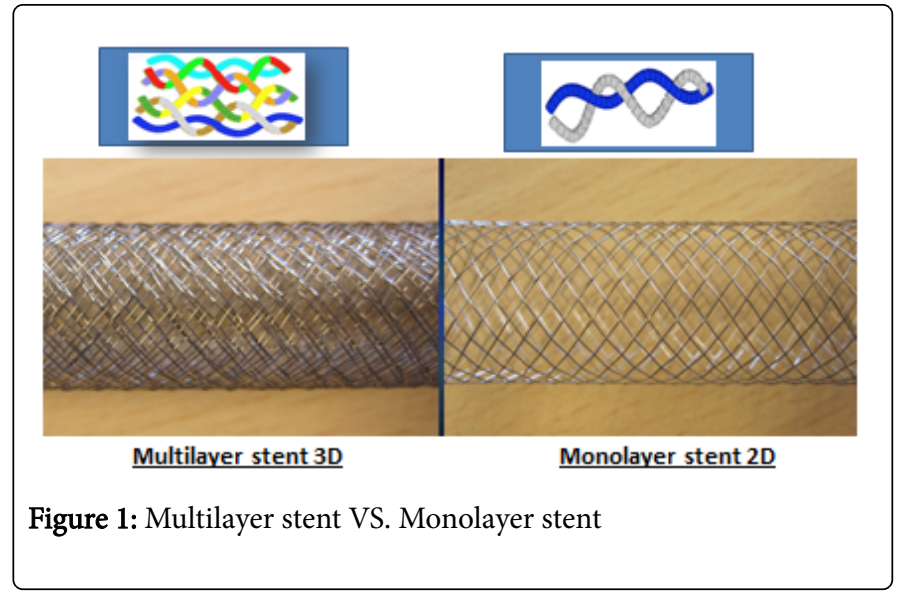

In saccular and fusiform aneurysms without collateral arteries, blood flow is swirling inside the sac. After the implantation of an MLS, the flow is redirected from the sac to the aortic lumen. This leads to a reduction of about $90 \%$ of the vortex, i.e., the swirling waves, which promotes the thrombus organization in several layers leading to a total thrombosis of the sac (Figure 2).

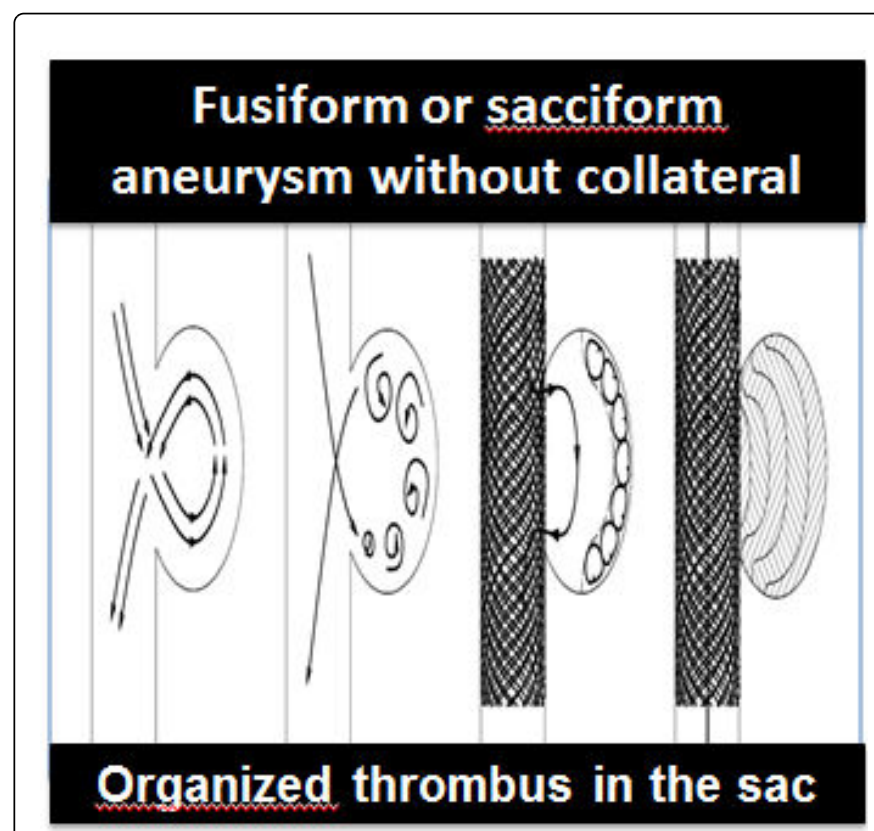

Figure 2: Concept of MLS in aneurysmal sac without collateral

In the presence of an important side branch out of the aneurismal sac, such as a renal artery, the flow is redirected to the side branch and becomes laminar. The aneurismal sac, no longer subject to the blood pressure constraints, will theoretically tend to shrink due to the Venturi effect (Figure 3). The shrinkage rate of the sac depends on the side branches diameter and the rigidity of the aneurysm walls.

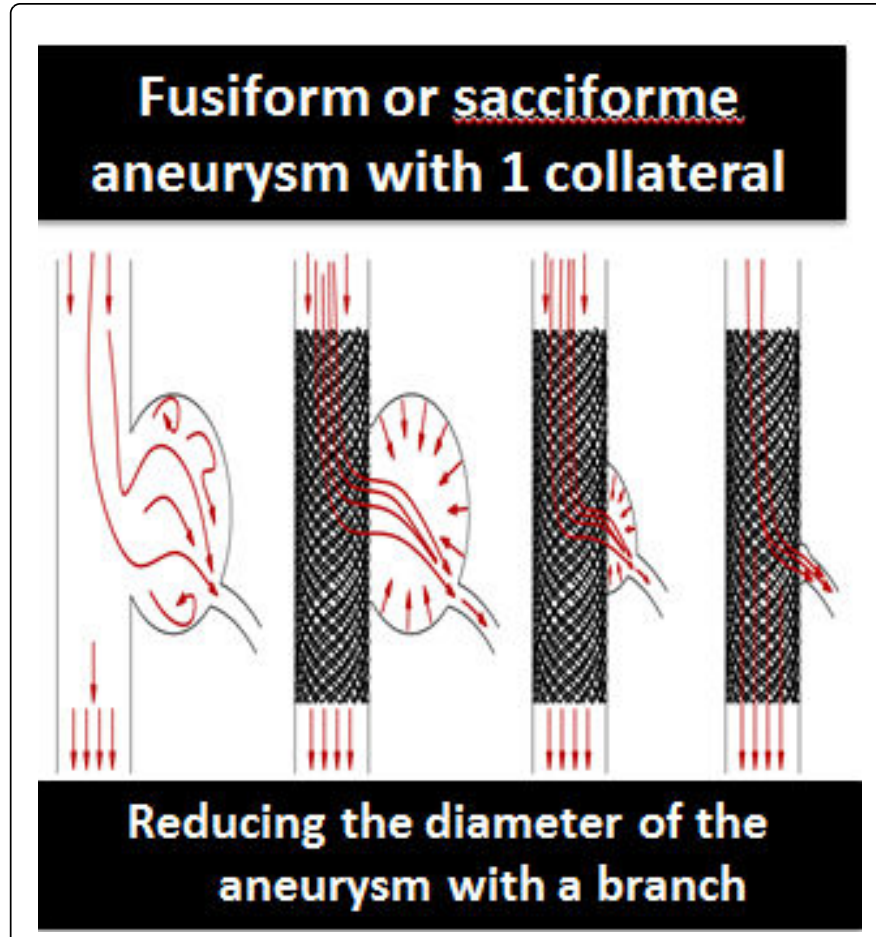

Figure 3: Concept of MLS in aneurysmal sac with collateral

\section{Materials and Methods}

The objective of the study was to conduct a monocentric prospective study to determine the efficacy, safety and performance of MLS and its delivery system in patients at high surgical risk presenting an aortic aneurysm and not suitable for currently available surgery. The inclusion criteria were age over 18 years, life expectancy $>12$ months, high surgical risk, ASA $<$ or $=3$, healthy proximal and distal landing zone, adequate arterial anatomy to perform procedure, healthy branches and collaterals (absence of stenosis or previously treated by angioplasty), aortic aneurysm (involving at least one branch in the aneurysm and/or in the landing zone), written informed consent signed by the patient. The non-inclusion were a ruptured aneurysm, medical contraindications to local or general anesthesia and angiography, congenital blood coagulation problems, intercurrent infection, or mycotic aneurysm, medical contraindications or allergy to all anticoagulant agents or anti- platelet drugs or aneurysm due to previously implanted stent graft. Were also excluded from the study the patients having prior surgical procedure within 30 days unless procedure is in preparation for device implantation or planned within 30 days post stent deployment, having had either a myocardial infarction or stroke within 6 weeks, renal insufficiency if creatinemia $>200 \mathrm{micromol} / \mathrm{I}$ before injection of contrast product and morbid obesity.

Post-operative control is organized according to a precise timetable at 1 month, 3 months, 6 months and then once a year. It is based on a clinical examination and radiological assessments: chest X-ray, abdomen without preparation, Doppler ultrasound and CT scan.

Primary endpoint is prevention of rupture, preservation of branches and collaterals, stabilization and exclusion of the aneurysm. The secondary endpoints are technical success in placement of the MLS, 
Page 3 of 6

the primary patency of the MLS and the occurrence of serious adverse events.

From March 2012 to December 2013, 41 procedures were performed on 41 aortic lesion locations in 38 patients treated by MLS for complex aortic pathologies.

Three patients are excluded from the study. Among them, 2 patients with large aneurysms ruptured and contained, classified Crawford IV respectively measuring $74 \mathrm{~mm}$ and $113 \mathrm{~mm}$ long axis, for which only MLS therapeutic option was possible due to the high surgical risk. These two patients died by a secondary rupture at 2 months and 3 months respectively. The 3 rd patient had a dissecting aneurysm classified Crawford II with a major diameter of $87 \mathrm{~mm}$. He died on the first postoperative day (D1) of a massive mesenteric infarction in relation with the overlapping of 3 MLS in the visceral aorta.

Our study covers only 38 patients because due to the many exclusion criteria and also, the low prevalence of thoraco-abdominal aortic aneurysm in Algeria. The study is purely descriptive and results are discussed in terms of frequency and percentages.

\section{Results}

\section{Patients' description}

The series of 38 patients selected for analysis includes 25 men and 13 women with an average age of 66.5 years [ $40-84$ years]. The patients had several comorbidities (Table 1).

\begin{tabular}{|l|l|l|l|l|l|l|l|}
\hline HBP & $\begin{array}{l}\text { Heart } \\
\text { disease }\end{array}$ & $\begin{array}{l}\text { Smokin } \\
\mathbf{g}\end{array}$ & COPD & CRF & Stroke & $\begin{array}{l}\text { Diabete } \\
\text { s }\end{array}$ & $\begin{array}{l}\text { Aortic } \\
\text { and / or } \\
\text { iliac } \\
\text { lesions }\end{array}$ \\
\hline $29 / 38$ & $6 / 38$ & $17 / 38$ & $5 / 38$ & $7 / 38$ & $3 / 38$ & $2 / 38$ & $8 / 38$ \\
\hline $76.3 \%$ & $15.8 \%$ & $44.7 \%$ & $13.2 \%$ & $18.4 \%$ & $7.9 \%$ & $5.3 \%$ & $21 \%$ \\
\hline
\end{tabular}

Table 1: Comorbidities of the study population; HBP: High Blood Pressure; COPD: Chronic Obstructive Pulmonary Disease; CRF: Chronic Renal Failure

The 41 procedures were divided into 4 groups (Table 2). It should be noted that 3 patients were treated for a double localization including 2 patients who had a lesion of the abdominal aorta associated with another thoracic aorta lesion. The third patient has a lesion in the aortic arch and in the descending thoracic aorta.

\begin{tabular}{|l|l|l|l|}
\hline Group 1 & TAAA / TAA & 21 cases & $51.2 \%$ \\
\hline Group 2 & AAA juxta /infra renal & 7 cases & $17.1 \%$ \\
\hline Group 3 & False aneurysm (FA) & 5 cases & $12.2 \%$ \\
\hline Group 4 & $\begin{array}{l}\text { Aortic dissecting hematoma (subgroup } \\
\text { 1:six cases)and extensive aortic } \\
\text { dissection(subgroup 2:two cases) }\end{array}$ & 8 cases & $19.5 \%$ \\
\hline
\end{tabular}

Table 2: Distribution of 41 aortic locations treated in 4 groups. TAAA: Thoraco-Abdominal Aortic Aneurysm; TAA: Thoracic Aortic Aneurysm; AAA: Abdominal Aortic Aneurysm

The first group includes 21 cases with 20 thoraco-abdominal aneurysms classified Crawford type I: 2 cases, type II: 4 cases, type III: 4 cases, type IV: 10 cases and 1 aneurysm of all descending thoracic aorta. The average diameter of these aneurysms was $71 \mathrm{~mm}$ (54-98 $\mathrm{mm})$.

The second group includes 7 cases of which 6 were juxta renal aneurysms and 1 infra renal aneurysm extended to the two common iliac arteries bifurcation. The average diameter of these aneurysms was $73 \mathrm{~mm}[62-97 \mathrm{~mm}]$.

The third group includes 5 cases of false aneurysm (FA) whose locations and origin are described on (Table 3).

\begin{tabular}{|l|l|l|}
\hline Location & origin & $\mathbf{N}$ (patient) \\
\hline Juxta renal & Tuberculosis & 1 \\
\hline Low thoracic aorta & Behcet's disease & 1 \\
\hline Aortic arch & Tuberculosis & 1 \\
\hline $\begin{array}{l}\text { Multiples: visceral aorta, Infra renal, } \\
\text { LCIA }\end{array}$ & Behcet's disease & 1 \\
\hline Aortic isthmus & Unspecified & 1 \\
\hline
\end{tabular}

Table 3: Aortic locations and origins of the false aortic aneurysms; LCIA: Left Common Iliac Artery

The forth group includes two sub groups. The first sub-group includes 6 cases of aortic dissecting hematoma, one located on the aortic arch and the 5 other located on the descending thoracic aorta. The second sub-group includes 2 complex cases: an extended non-A non-B dissection associated with an aberrant right subclavian artery in one case and downstream of the distal anastomosis of an aorto-aortic prosthesis initially set for the treatment of an aortic dissection Stanford type $\mathrm{A}$ in the other case.

All the MLS implantation procedures have been performed in the operating room with the same preparation as conventional surgery and after systemic heparinization of $0.5 \mathrm{mg} / \mathrm{kg}$.

The procedure was performed under local anesthesia in 3 cases (8\%), loco regional in $19(50 \%)$ and general in 16 cases (42\%). The surgical approach was the common femoral artery except in two cases. For one patient, a common iliac artery approach was necessary due to the obstruction of the two external iliac arteries. For another patient with aorto-bi-iliac obstruction, the axillary approach was used.

The total average fluoroscopic time exposure was of $38 \mathrm{~min}$ (12-90 $\mathrm{min})$. The average duration of intervention was $80 \mathrm{~min}$ (20-165 min). The average duration of the MLS deployment was in average $3 \mathrm{~min}$ (1-4 $\mathrm{min}$ ). In 7 cases, revascularization procedures have been associated with the implantation of the MLS: 2 cross over bypasses femoro-femoral, 1 ilio- femoral bypass and 4 cases of iliac angioplasty.

\section{Patients follow up}

There were 3 cases of complications related to access which have been treated surgically with success. No surgical conversion was performed.

Technical success was $100 \%$. All visceral arteries remained patent at the angiography control. There were 3 endoleaks (7.3\%) including 2 cases (type I and type III) diagnosed intra operatively were immediately corrected by an additional MLS. A third case (endoleak type I) was diagnosed at the one month control. It was treated by an additional MLS. 
Citation: Bouayed MN, Bouziane L, Sekkal A, Benaroussi SA (2016) Treatment of Complex Aorta Diseases by Multilayer Stents. J Vasc 2: 111.

Page 4 of 6

There were two heart complications including a massive lethal myocardial infarction. One patient died from an aneurysm rupture at day 0 . The 30 -day mortality is therefore $5 \%$.

Two patients had renal failure, one among them required dialysis (5\%). Neither paraplegia nor mesenteric ischemia has been observed.

The average hospital stay was 7 days $(4-14$ D). Treatment at discharge included the association of two antiplatelets and a statin in addition to the control of risk factors and the specific treatment in the event of Behcet's disease.

The mean follow-up was 12 months (1-20 months). There has been 9 deaths (25\%) of late mortality not linked to aneurysm, occurring among older patients and with several co morbidities.
There was no aneurismal rupture or migration of the MLS. The aneurismal sac is not supplied in 14 locations (39\%), low supplied in 19 locations $(50 \%)$ and 3 locations $(8 \%)$ recently treated are still under study (Table 4). The best results were obtained in FA and in aortic dissecting hematoma whose exclusion was fast, occurring in 1.5 months on average [1-3 months].The two complex extensive dissections remained stable without aneurismal evolution. Visceral arteries remained patent in $100 \%$ of the cases (Figures 4 and 5).The evolution of false aneurysms was quickly remarkable (Figures 6a, 6b and $7 a, 7 b)$.

\begin{tabular}{|l|l|l|l|l|l|l|l|l|}
\hline \multicolumn{2}{|l}{ Groups } & N & \multicolumn{2}{l|}{ Not supplied } & \multicolumn{2}{l|}{ Low supplied } & \multicolumn{2}{l|}{ Supplied (no change) } \\
\hline 1 & TAAA/TAA & 20 & $6 / 20$ & $30 \%$ & $14 / 20$ & $70 \%$ & 0 & $0 \%$ \\
\hline 2 & AAA juxta / infra renal & 6 & $3 / 6$ & $50 \%$ & $2 / 6$ & $33 \%$ & 1 Recent & 0 \\
\hline 3 & False aneurysms & 4 & $3 / 4$ & $75 \%$ & $1 / 4$ & $25 \%$ & $0 \%$ & 2 Recent \\
\hline 4 & Aortic dissecting hematoma & 6 & $2 / 6$ & $33 \%$ & $2 / 6$ & $33 \%$ & & \\
\cline { 2 - 9 } & Extensive aortic dissection & 2 & $\begin{array}{l}\text { Stable, } \\
\text { absence of } \\
\text { aneurysm }\end{array}$ & & & & & \\
\end{tabular}

Table 4: Evolution of the sac according to the treated groups, Followed by 36 patients ( 38 treated aortic locations)
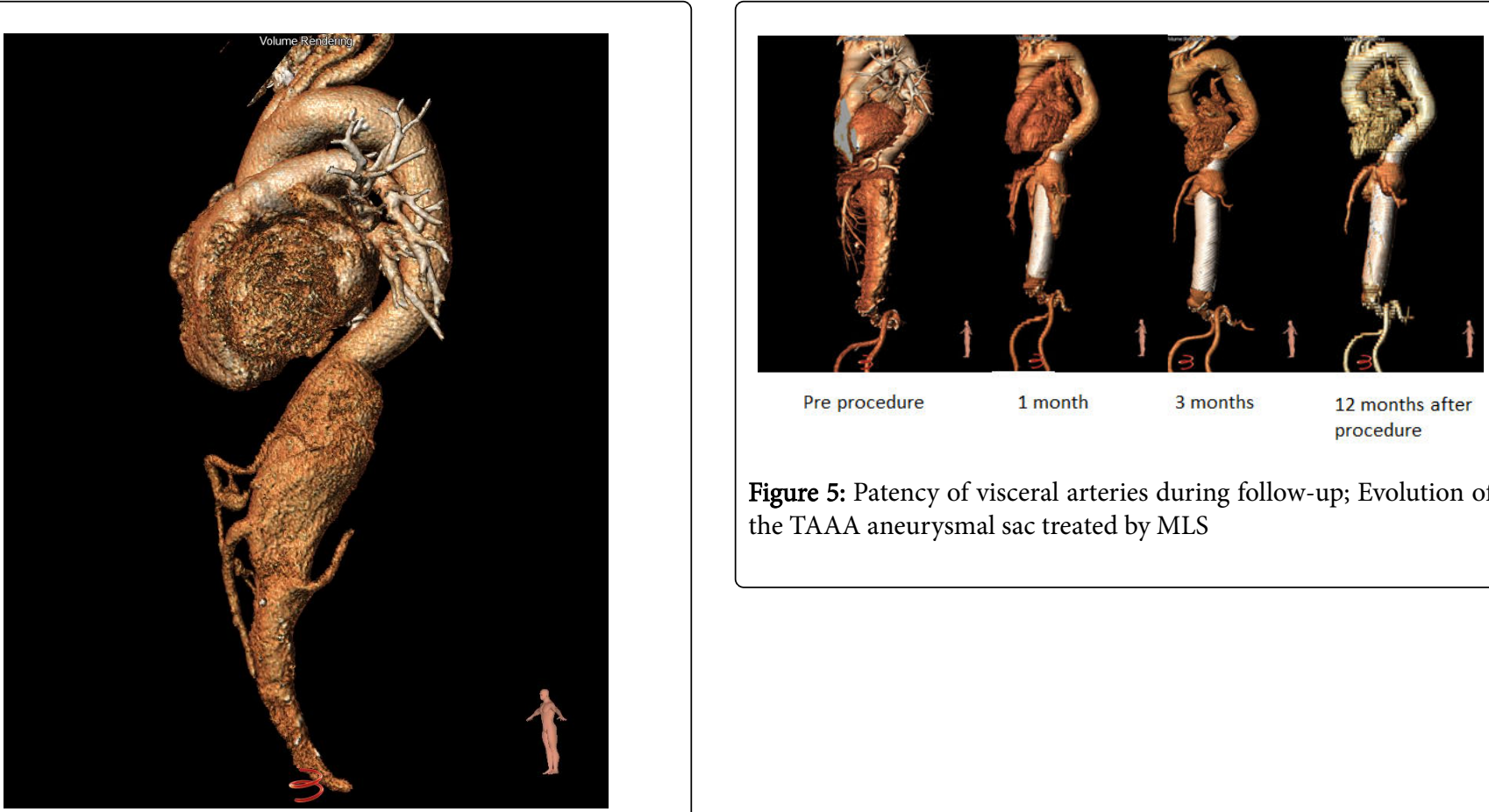

Figure 5: Patency of visceral arteries during follow-up; Evolution of the TAAA aneurysmal sac treated by MLS

Figure 4: Fusiform aneurysm / Crawford type IV Maximum Diameter $81 \mathrm{~mm}$ 

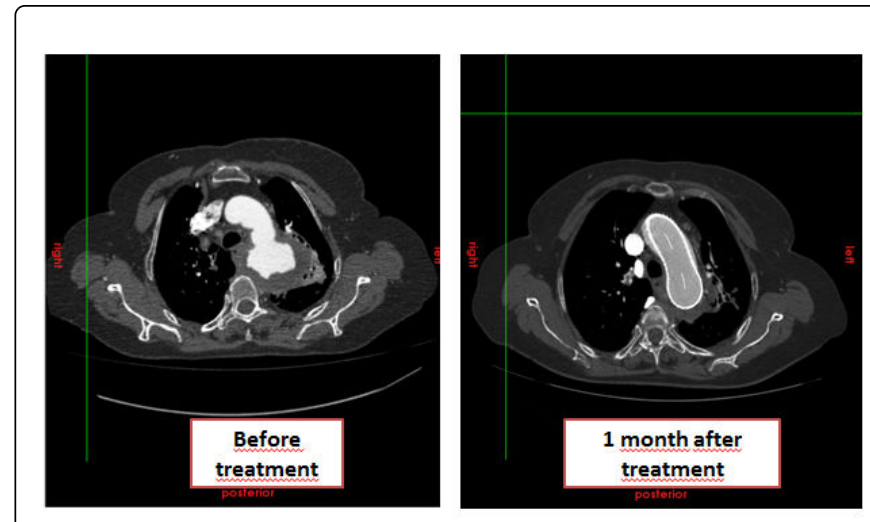

Figure 6a: Evolution of tuberculosis false aneurysm sac treated by MLS (axial section)

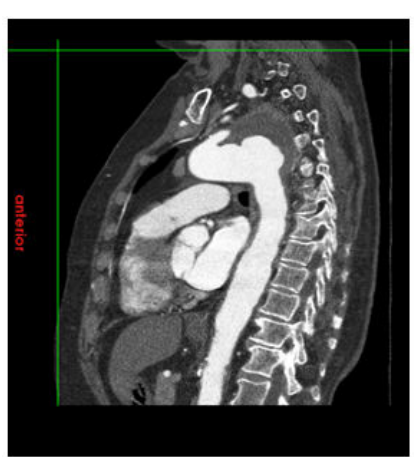

Before treatment

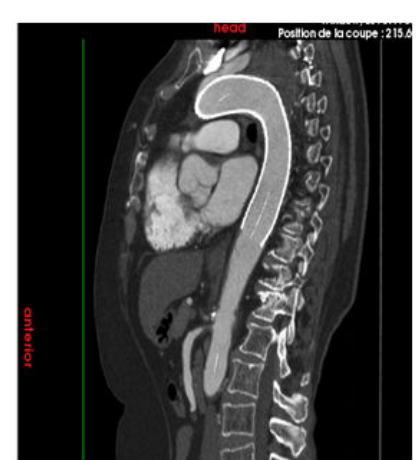

1 month after treatment
Figure 6b: Evolution of tuberculosis false aneurysm sac treated by MLS (sagittal section)
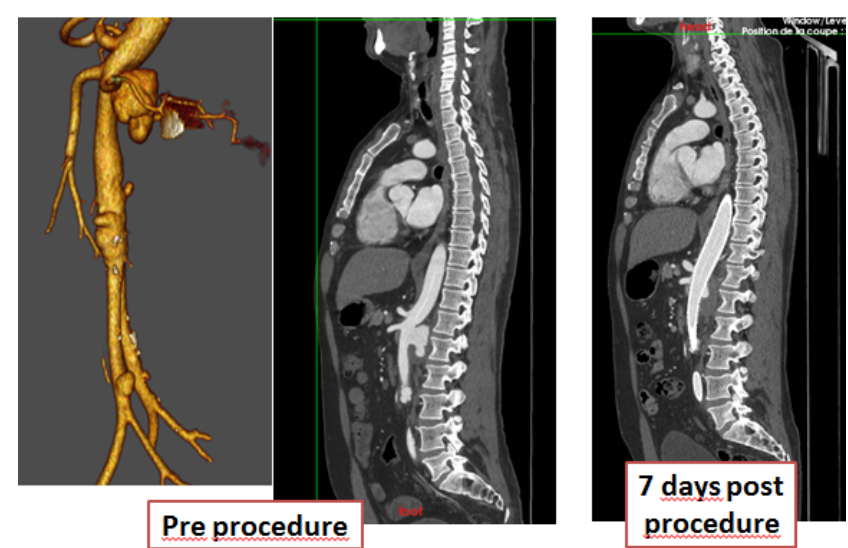

Figure 7a: Aorta visceral false aneurysm secondary to Behcet's disease (3D and sagittal sections)

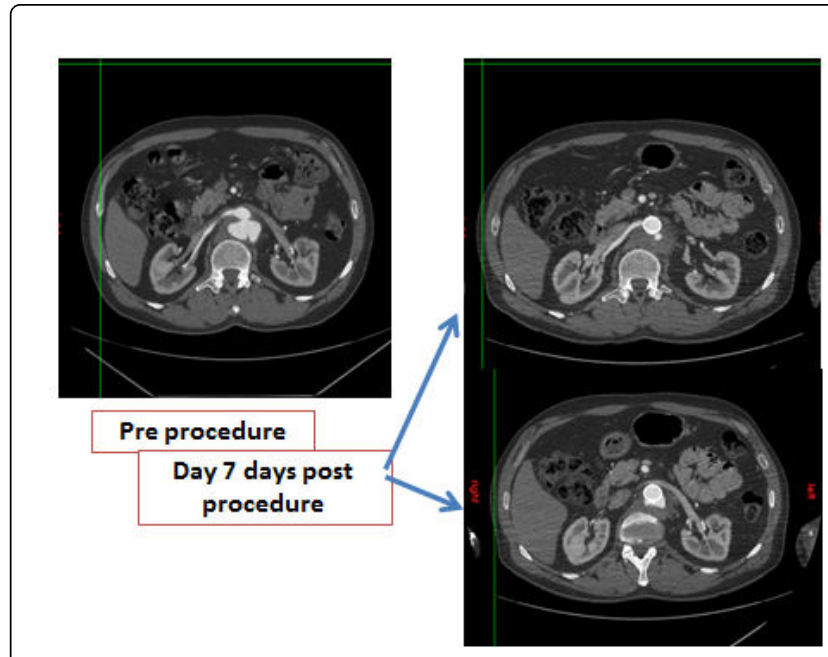

Figure 7b: Aorta visceral false aneurysm secondary to Behcet's disease

\section{Discussion}

The management of aortic diseases including aneurysms, dissections and false aneurysms has been completely reconsidered in recent years due to development of endovascular techniques and covered endografts (CE). The TAA is an indication of choice for a stent graft but if it is extended to visceral arteries or supra-aortic trunks (SAT), its treatment becomes difficult. For the lesions of the aortic arch, endovascular treatment is less invasive than classic surgery which uses extra-corporeal circulation and deep hypothermia especially as it often addresses frail patients. However, the problem at this level is more complex because it must take into account the angulations of the aortic arch, the extent of lesions and the reports of the ostial SAT within these lesions. So more complex endovascular treatments are necessary (chimney, periscope, transposition techniques first of SAT prior to the establishment of a CE) [4].

The problem remains also for the TAAA due to the need to preserve the permeability of the medullar arteries and visceral arteries. In effect, paraplegia is a major complication of vascular surgery and even for the treatment by $\mathrm{CE}$ which is accompanied by a rate that varies from 1 to $6 \%$ [4]. Conventional surgical treatment of TAAA is accompanied by relatively high mortality and morbidity. According to the American Registry, Cowan found a rate of hospital mortality of $22.3 \%$ [5]. The literature reports a rate of post-operative mortality ranging from 7 to $35 \%$ and a rate of paraplegia ranging from 4 to $21 \%$. The best results are obtained in specialized centers mastering the treatment of TAAA, where the frequency of this type of intervention is important.

The hybrid treatment is also not devoid of risks. Chiesa reports on a series of 49 patients treated for TAAA by hybrid methods with a mean follow-up of 21.3 months, 4 deaths including 3 of an aortic rupture and 1 of a visceral ischemia secondary to a thrombosis of the visceral prosthesis [6]. Kabbani reports a series of 36 patients whose average age is 71 years, with an antero-posterior diameter aneurysm average of $63 \mathrm{~mm}$. His sample of patients included:1 TAAA Crawford type I, 10 type II, 12 type III, 10 type IV and 3 para renal aneurysms. Four patients were symptomatic. 123 bypasses for visceral artery revascularization have been performed, with an average of three per 
patient. Retrograde revascularization was done in 30 patients and anterograde in 3 patients. The hospital mortality was $8.3 \%$. There was 1 paraparesis, $46 \%$ of severe post-operative complications, 14 endoleaks and an average of relatively long stay of 26 days [7].

Sonesson reported a series of 7 patients of TAAA treated by FBE. He estimated that surgery gave a higher mortality rate than endovascular treatment. This rate was $19 \%$ at 30 days and $31 \%$ at one year in the surgical treatment against $0 \%$ in the endovascular treatment with a mean follow-up of 7 months (3-16 months). But morbidity of FBE treatment was severe since there were 2 cases of mesenteric ischemia and 3 cases of paraplegia [8]. The treatment by FBE has many disadvantages. Roselli study results from a series of 73 patients showed $5.5 \%$ mortality, $4 \%$ of medullar complications and $11 \%$ of acute renal failure [9].

A multicenter study has been carried out by the University Association of Research in Vascular Surgery (AURC) over a period ranging from May 2004 to January 2009 on a series of 134 patients with an average age of 73 years (48-91 years) who had an endovascular treatment by fenestrated endografts. The antero-posterior diameter aneurysms ranged between 45 and $91 \mathrm{~mm}, 403$ visceral arteries were revascularized including 256 renal arteries. The 30-day mortality was $2.2 \%$ (3 patients out of 134 ), with a paraplegia rate of $0,7 \%$ ( 1 patient out of 134) and $1.4 \%$ (2 patients out of 134) of postoperative permanent dialysis. There have been 16 cases of endoleaks. For a mean follow-up of 15 months (2-53 months), the survival at 12 months was $75 \%$. In this cohort, 12 patients required revision surgery [4].

FBE treatment has its own limits. Custom made stent grafts are expensive and have a manufacturing deadline. The off-the-shelf multibranch stent grafts are under evaluation. These FBE are wide and may not be used when the access is itself limited by the calcifications and tortuosity. They may not be used when the visceral arteries have a diameter of less than $4 \mathrm{~mm}$. In addition their rate of patency and longterm durability are not yet clearly specified.

Our study shows, for the moment, an increased safety with the use of MLS. Indeed, in our cohort, 38 patients were accumulating several risk factors, exhibited in more than half of the cases of TAAA and complex lesions due to dual localization as well as lesions of the aortic arch.

More than half of the procedures were performed under regional or local anesthesia with radiation and intervention duration relatively short. Most often no blood transfusion was necessary. Calcifications and tortuosity do not formally contraindicate the access. In addition, it is possible to choose an alternative access in the event of bilateral iliac occlusion. The absence of paraplegia gives MLS significant advantage. Furthermore, there was no cerebral stroke or mesenteric ischemia. The hospital stay was short, 7 days on average. The 30 day mortality is low: 1 case not linked to the aneurysm and 1 case in relation to the aneurysm. The late mortality unrelated with the aneurysm was $24 \%$ ( 9 deaths) and $0 \%$ in relation to the aneurysm. The deaths have impacted the most elderly patients with multiple co-morbidities.

On a mean follow up of 12 months, the visceral arteries and SAT remained patent in $100 \%$ cases.

In our study, results showed that MLS are effective in treating false aneurysms with complex localization, including aneurysms without significant collateral. For the remaining, studies are on-going and results accuracy can only be achieved on a very long term and large sample.

Therefore, MLS have many benefits. However, good results cannot be expected without a strict compliance to certain technical requirements: oversizing of $20 \%$ in case of an aneurysm and $10 \%$ in case of a dissection. It is necessary to observe a minimum of $3 \mathrm{~cm}$ landing zone and respect an overlapping MLS zone of 3 to $5 \mathrm{~cm}$ avoiding its placement at the level of visceral aorta. It is also recommended to plan a wide coverage of the aneurysm.

Treatment with MLS has some drawbacks to overcome such as the difficulty to treat visceral stenosis arteries after the insertion of the MLS, jump of the MLS, shrinkage of the MLS .We experimented these in some occasions during our study. Finally, it is to consider that the time it takes for the thrombus to fill the aneurismal sac is proportional to its diameter. The larger the diameter the longer the period, arising the issue of the treatment for large size aneurysms.

\section{Conclusion}

MLS may be a treatment option for dissections and complex aneurismal disease of the aorta in frail patients with several comorbidities. When an aneurysm has no large collateral or false aneurysm, MLS is effective in the prevention of rupture and therefore in the total exclusion of the aneurismal sac. However in true aortic aneurysms with large side branches, the judgment criteria (risk of rupture and mortality in relation with the aneurysm) can only be assessed on large series and on a long follow-up, superior to 5 years.

\section{References}

1. Augsburger L, Farhat M, Asakura F, et al. Hemodynamical effects of Cardiatis braided stents in sidewall aneurysm silicone models using PIV.

2. Bonneau $\mathrm{M}$, Kang C. Assessment of peripheral multilayer stent technology in pig-model aneurysm.

3. Wailliez C, Coussement G. CFD study of multilayer stent haemodinamics effects in abdominal aortic aneurysms.

4. Amiot S, Haulon S, Becquemin JP, Magnan PE, Lermusiaux P,et al. (2010) Fenestrated Endovascular Grafting: The French Multicentre Experience Eur J Vasc Endovasc Surg 39: 537-544.

5. Cowan Jr JA, Dimick JB, Henke PK, Huber TS, Stanley JC, et al. (2003) Surgical treatment of intact thoracoabdominal aortic aneurysms in the United states: hospital and surgeon volume-related outcomes. J Vasc Surg 37: 1169-1174

6. Chiesa R. (2012) Hybrid surgery for TAA is the answer. Abstracts book MEET. Rome: p 39.

7. Kabbani LS, Criado E, Upchurch GR Jr, Patel HJ, Eliason JL, et al. (2010) Hybrid repair of aortic aneurysms involving the visceral and renal vessels. Ann Vasc Surg 24: 219-224.

8. Sonesson B, Resch T, Dias N, Kristmundsson T, Malina (2010) Fenestrated and branched endografts for thoracoabdominal aneurysms: Nordic results.In: Becquemin JP, Alimi YS editors. Controversies and updates in vascular surgery. Turin: Edizioni Minerva Medi 315-320.

9. Roselli EE, Greenberg RK, Pfaff K, Francis C, Svensson LG, et al. (2007) Endovascular treatment of thoracoabdominal aortic aneurysms. J Thorac Cardiovasc Surg 133: 1474-1482. 